\title{
COENZYME Q10 DEFICIENCY AND TYPE 2C MUSCLE FIBERS
}

Investigators at Washington University School of Medicine, St Louis, MO, evaluated retrospectively clinical, laboratory, and muscle histochemistry and oxidative enzyme characteristics in 49 children with suspected mitochondrial disorders. Children $(n=18)$ with CoQ10 deficiency in muscle were compared to 31 with normal CoQ10 values. Motor delay/hypotonia and cognitive/language delay were the most frequent clinical features in CoQ10-deficient and control groups. Seizures or epileptiform EEG occurred in 56\%. Type $2 \mathrm{C}$ muscle fibers were 5.5 -fold more frequent in CoQ10-deficient patients compared with mitochondrial and nonmitochondrial controls $(\mathrm{P}<0.0001)$. Type $2 \mathrm{C}$ fiber frequency of $>5 \%$ had $89 \%$ sensitivity and $84 \%$ specificity for CoQ 10 deficiency in these patients. No biopsy showed active myopathy. Multiple abnormalities in muscle oxidative enzyme activities were more frequent in CoQ10-deficient patients than controls. (Sommerville RB, Zaidman CM, Pestronk A. Coenzyme Q10 deficiency in children: Frequent type 2C muscle fibers with normal morphology. Muscle Nerve 2013 Nov;48(5):722-6). (Response: RB Sommerville; E-mail: sommervileb@neuro.wusti.edu).

COMMENT. An increased frequency of type $2 \mathrm{C}$ fibers in morphologically normal muscle is a sensitive and specific histological marker of CoQ10 deficiency in a child suspected of having a mitochondrial disorder. Clinical syndromes associated with CoQ10 deficiency are diverse and include myopathy, cerebellar ataxia, nephrotic syndrome, and encephalopathy including Leigh syndrome. Early diagnosis is important since some cases are responsive to treatment with $\mathrm{CoQ} 10$ supplementation.

\section{NEUROPHYSIOLOGICAL TESTING IN NORMAL CHILDREN}

Investigators in Belgrade, Serbia, and Newcastle upon Tyne, UK, reviewed the literature (Ovid Medline, 1948-2012) for citations on repetitive nerve stimulation (RNS) and single fiber electromyography (SFEMG) in children. Five articles contained data on 48 normal children studied with RNS and only 1 article with data on SFEMG in 20 normal children were located. Significant differences in the response to RNS in children compared to adults were identified, emphasizing the need for age appropriate normal values. (Kosac A, Gavillet E, Whittaker RG. Neurophysiological testing in congenital myasthenic syndromes: a systematic review of published normal data. Muscle Nerve 2013 Nov;48(5):711-5). (Response: RG Whittaker; e-mail: roger.whittaker@ncl.ac.uk).

COMMENT. The late Dr Ingrid Gamstorp published extensively on normal conduction velocities of peripheral nerves in infancy, childhood, and adolescence: Acta Paediatr Scand Suppl 1963 Jul;52(S146):68-76; Acta Paediatr Scand 1965 Jul;54(4):309-13. Also, in Oh SJ. Clinical Electromyography: Nerve Conduction Studies. $3^{\text {rd }}$ ed. Philadelphia: Lippincott William \& Wilkins; 2003. 848 p.; Dr Gamstorp's sensory nerve conduction research was conducted while a visitor in the Division of Neurology, 1964-5, at then Children's Memorial, now Ann \& Robert H. Lurie Children's Hospital of Chicago. 